\title{
RESULTADOS DO ESTUDO DA DISPENSAÇÃO DE MEDICAMENTOS NO SERVIÇO DE FARMÁCIA DO HOSPITAL DISTRITAL GONZAGA MOTA DE MESSEJANA - CEARÁ
}

\author{
Results of study of the dispensation of medicines in the pharmacy service of \\ district hospital Gonzaga Mota of Messejana - Ceará \\ Elisângela P. Silva ${ }^{1}$, Liduína F. P. Barreto ${ }^{2}$, Rivelilson M. Freitas ${ }^{3 *}$ \\ ${ }^{1}$ Estagiária do Hospital Distrital Gonzaga Mota de Messejana - (HDGMM) - Fortaleza - CE e Acadêmica do Curso \\ de Farmácia da Faculdade Católica Rainha do Sertão. \\ ${ }^{2}$ Chefe do Serviço de Farmácia e Farmacêutica Hospitalar do HDGMM; \\ ${ }^{3]}$ Farmacêutico Hospitalar do HDGMM e Coordenador do Curso de Farmácia da Faculdade Católica Rainha do \\ Sertão.
}

*Autor para correspondência e-mail: rivmendes@hotmail.com

Recebido em 07/03/2008- Aceito em 10/06/2008

RESUMO: No presente estudo avaliou-se a dispensação de medicamentos aos pacientes no serviço de farmácia do Hospital Distrital Gonzaga Mota de Messejana (HDGMM), em particular observou-se o perfil farmacoepidemiológico, as principais reações adversas, as hipóteses diagnósticas, os problemas relacionados a medicamentos e à saúde. Todos os pacientes atendidos eram adultos e à faixa etária mais prevalente foi a de 3150 anos, sendo $33 \%$ do sexo masculino e $67 \%$ do sexo feminino. A maioria (40\%) estudou somente até o ensino fundamental, eram casados ou tinham uma relação estável. Em relação as principal reação adversa foi vômitos (24\%), enquanto que 1411 (42\%) pacientes apresentaram como hipótese diagnóstica hipertensão arterial sistêmica e o tabagismo como principal problema relacionado à saúde (60\%). E entre os problemas relacionados aos medicamentos (PRM) o mais prevalente foi o PRM 6 (50\%), seguido do PRM 4 em 19\% das pacientes. Os dados obtidos indicam a necessidade de implantação de um serviço de atenção farmacêutica, para orientação dos pacientes e consequentemente melhora na aderência dos pacientes ao esquema terapêutico.

PALAVRAS-CHAVE: Atenção farmacêutica; promoção de saúde; serviço de farmácia hospitalar; dispensação.

ABSTRACT: In this study, to dispensing drugs to patients in the pharmacy department of the Hospital District of Messejana Gonzaga Mota (HDGMM), in particular there was the profile pharmacoepidemiological, the main adverse reactions, the diagnostic possibilities, the problems related to drugs and health. All patients were adults and age group was the most prevalent of $31-50$ years and $33 \%$ male and $67 \%$ female. The majority (40\%) studied only up to primary school, were married or had a stable relationship. For the main adverse reaction was vomiting (24\%), while $1411(42 \%)$ patients had diagnostic hypothesis as hypertension and smoking as the main problem related to health (60\%). Among the problems related to drugs (DRP) the most prevalent was the DRP $6(50 \%)$, followed by the DRP 4 in 19\% of patients. These data indicate the need for deployment of a service of pharmaceutical care, for the guidance of patients and thus improves the adherence of patients to therapeutic scheme.

KEYWORDS: Pharmaceutical care, promotion of health; service hospital pharmacy; dispensation.

\section{INTRODUÇÃO}

A Atenção Farmacêutica, entendida como um modelo de prática profissional desenvolvida no contexto da assistência farmacêutica, de acordo com a proposta do Consenso Brasileiro de Atenção Farmacêutica, possui por finalidade aumentar a efetividade do tratamento medicamentoso, concomitante à deteç̧ão de problemas relacionados a medicamentos (MONTRUCHIO et al., 2005). 
A Atenção Farmacêutica contempla a interação direta com os pacientes, configurando uma opção mais avançada para o pleno exercício da profissão farmacêutica, pois, além de permitir a aplicação dos conhecimentos acumulados, promove a integração com médicos, enfermeiros, nutricionistas e aos demais profissionais de saúde, assegurando o uso correto do medicamento e evitando o aparecimento de reações adversas, toxicidade e interações medicamentosas (HEPLER \& STRAND, 1999).

A complexidade das terapias medicamentosas e as evidências dos resultados das intervenções farmacêuticas na melhoria dos regimes terapêuticos e na redução dos custos assistenciais reforçam a importância de uma assistência farmacêutica de qualidade (HEPLER \& STRAND, 1999). O serviço de farmácia tem participação importante na elaboração de uma política de uso racional de medicamentos, visando melhorar e garantir a qualidade da farmacoterapia e reduzir os custos para o estabelecimento, já que a politerapia, além de onerar os custos com cuidado ao paciente, ocasiona elevação da morbi-mortalidade (BARR, 1955; STELL, et al., COUCH, et al., 1981; FLEMING, 1996; BATES, et al., 1998; CIPOLLE, et al., 2002).

O papel do farmacêutico dentro do contexto hospitalar deixou de ser apenas administrativo, na programação de medicamentos e organização dos recursos financeiros. A tendência atual é que a atenção farmacêutica direcione-se para o paciente, tendo o medicamento como instrumento e não mais como meio (GOMES \& REIS, 2001). Desta forma, promove suporte técnico-científico junto à equipe de saúde, na análise de prescrição, monitorização do tratamento e do quadro clínico do paciente durante a sua internação (GOMES \& REIS, 2001; HEPLER \& STRAND, 1999; BRASIL, 1994), porém a atividade do farmacêutico não finaliza com a alta do paciente. $\mathrm{Na}$ alta do paciente, comumente há prescrição de medicamentos, portanto o farmacêutico deve orientá-lo, mesmo quando não há necessidade de continuar a terapia, é necessário educá-lo, para que passe a ver o medicamento não como um bem de consumo, mas como um instrumento útil, capaz de prevenir a doença e restaurar a saúde por uma utilização adequada (HEPLER \& STRAND, 1999; BRASIL, 1994).

A metodologia atual para o desenvolvimento desse trabalho clínico do farmacêutico é a Atenção Farmacêutica, mudança filosófica originada no início dos anos 90 definida como: "Provisão responsável pela terapia medicamentosa com o propósito de alcançar resultados definidos que melhore a qualidade de vida do paciente. Esses resultados são: cura da doença, eliminação ou redução dos sintomas, diminuição da velocidade da progressão da doença, e prevenção da doença ou de seus sintomas" (HEPLER \& STRAND, 1999).

Nesse contexto, a prática da Atenção Farmacêutica Hospitalar está sendo implantada no Serviço de Farmácia Hospitalar do Hospital Distrital Gonzaga Mota de Messejana (Gonzaguinha de Messejana), por meio de intervenções na emergência desta unidade que tem como objetivo a implantação desta filosofia dirigida aos pacientes.

O Gonzaguinha de Messejana, localizado em Fortaleza - Ceará foi fundado em 1986, e é mantido pela Prefeitura Municipal de Fortaleza. É considerado uma das principais unidades de atendimento de Messejana pelo Sistema Único de Saúde (SUS). Possui atualmente capacidade de 100 leitos, oferecendo atendimento em diversas clínicas, maternidade e emergência.

Diante deste contexto, foi realizada a análise dos dados coletados dos pacientes acompanhados na emergência do referido estabelecimento com objetivo de verificar dados sócio-econômicos, principais doenças, problemas de saúde, os principais medicamentos prescritos e os problemas relacionados a estes.

\section{MATERIAL E MÉTODOS}

Os acompanhamentos seguiram a metodologia proposta pelo Programa Dáder (CIPOLLE, et al., 2002; GRUPO DE INVESTIGACIÓN EN ATENCIÓN FARMACÉUTICA, 2002; THE UNITED STATES PHARMACOPOEIA, 2002; FERNANDEZ-LLIMÓS, et al., 1999).

Foram considerados problemas reais e risco de PRM (quando o paciente apresenta fatores de risco que podem desencadeá-lo) e também os problemas de saúde caracterizados como aqueles não relacionados ao medicamento e que necessitam de intervenção, conforme preconizado pela Organização Mundial de Saúde (FERNANDEZ-LLIMÓS, et al., 1999; ORGANIZAÇÃO MUNDIAL DE SAÚDE, 1995).

Os resultados foram obtidos por meio de um estudo retrospectivo observacional dos acompanhamentos realizados no Gonzaguinha de Messejana no período de Setembro de 2006 à Setembro de 2007.

\section{Coleta dos dados}

A coleta dos dados dos pacientes foi realizada pelos acadêmicos de Farmácia da Faculdade Católica Rainha do Sertão, treinados sob supervisão, sendo realizada por meio de consulta aos prontuários que continham as seguintes informações retiradas do prontuário médico e da entrevista farmacêutica com o paciente: idade, sexo, patologias pregressas, história medicamentosa pregressa, dados clínicos do paciente como hipótese diagnóstica (considerada a doença que levou o paciente à internação), outras doenças apresentadas, os 
medicamentos prescritos, procedimentos médicos de relevância realizados no período de internação, problemas relacionados com medicamentos e detecção de riscos para desenvolvê-los. Não houve identificação nominal, nem risco moral para os pacientes, por se tratar apenas de dados estatísticos.

\section{Análise dos dados}

As informações coletadas foram lançadas em sistema informatizado Excel ${ }^{\circledR}$, versão 2003, para análise dos dados em percentagem e elaboração dos gráficos.

\section{RESULTADOS E DISCUSSÃO}

Foi acompanhado um total de 3360 pacientes, com uma média mensal de 280 referentes aos atendimentos de emergência, apresentando uma faixa etária predominante de 31 e 50 anos (45\%) (Figura 1). Para os resultados quanto ao sexo o de maior prevalência foi o do sexo feminino $(67,0 \%)$.

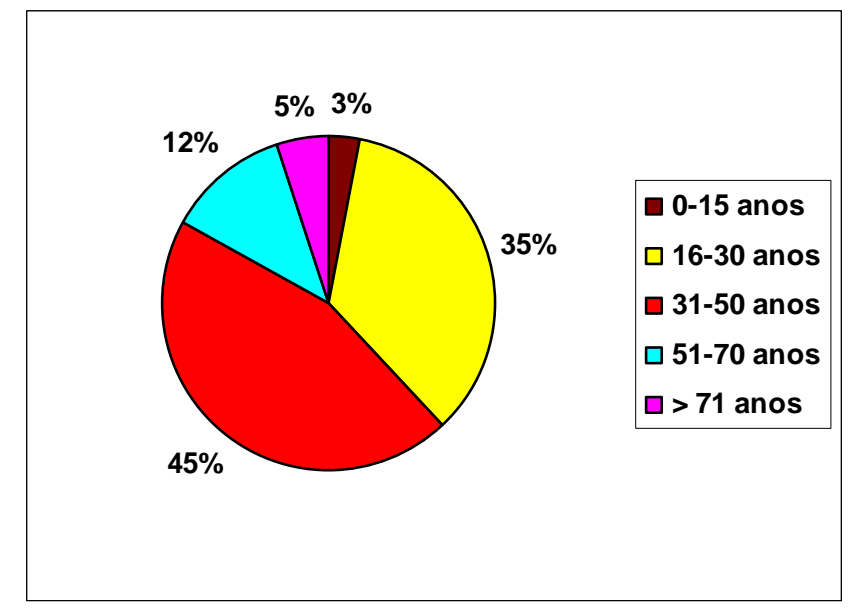

Figura 1: Idade dos pacientes acompanhados no Hospital Gonzaguinha de Messejana.

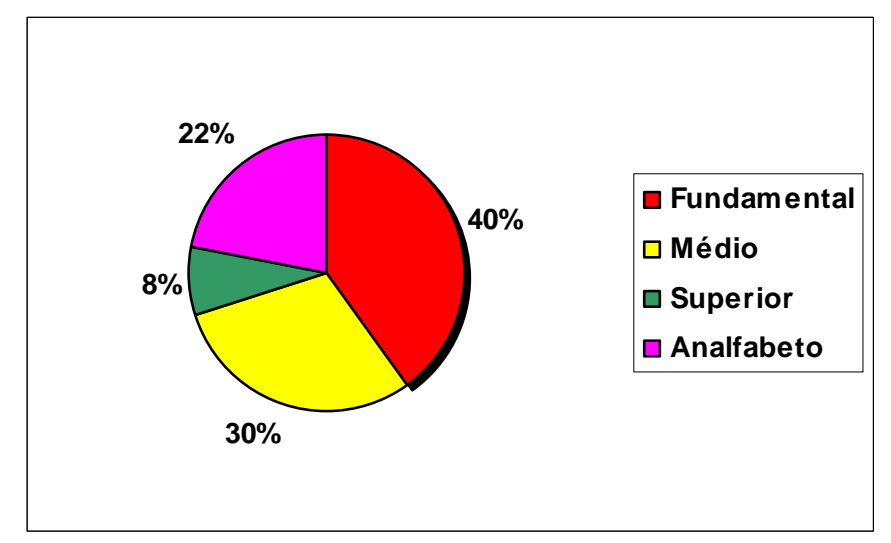

Figura 2: Distribuição da escolaridade entre os pacientes acompanhados no Hospital Gonzaguinha de Messejana. 


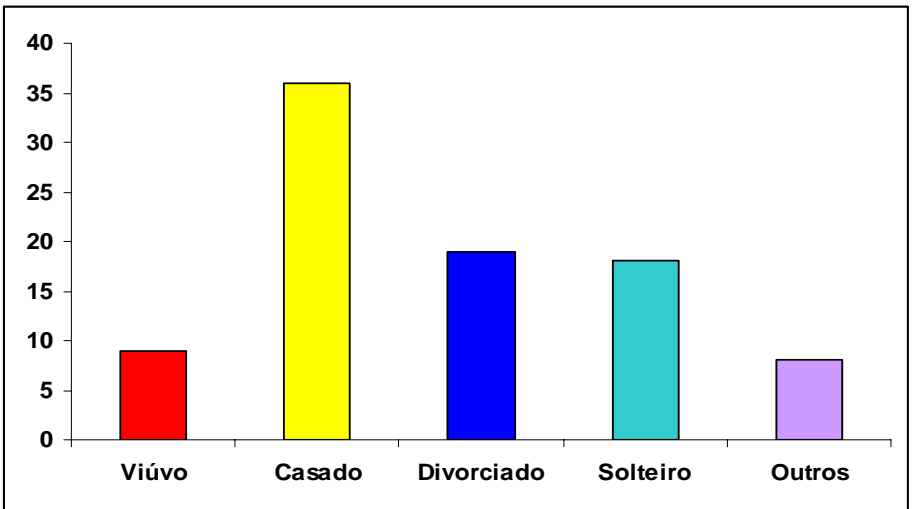

Figura 3: Distribuição do estado civil entre os pacientes acompanhados no Hospital Gonzaguinha de Messejana.

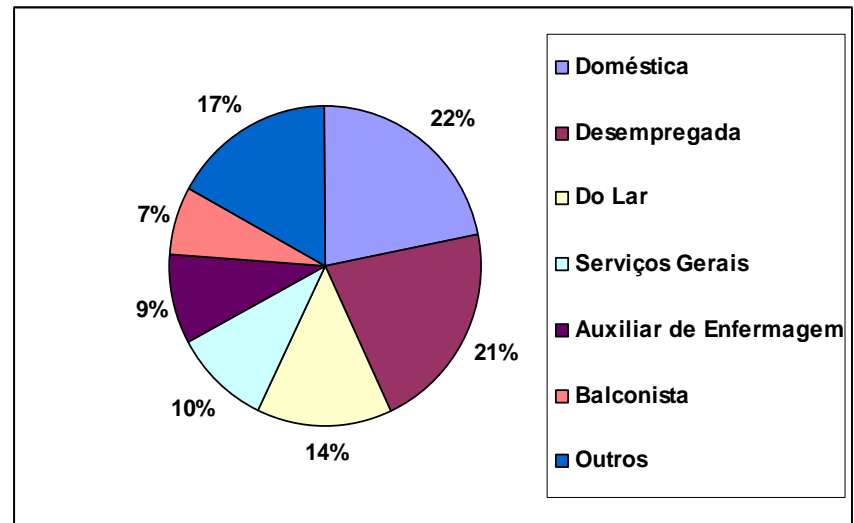

Figura 4: Distribuição da profissão entre os pacientes acompanhados no Hospital Gonzaguinha de Messejana.

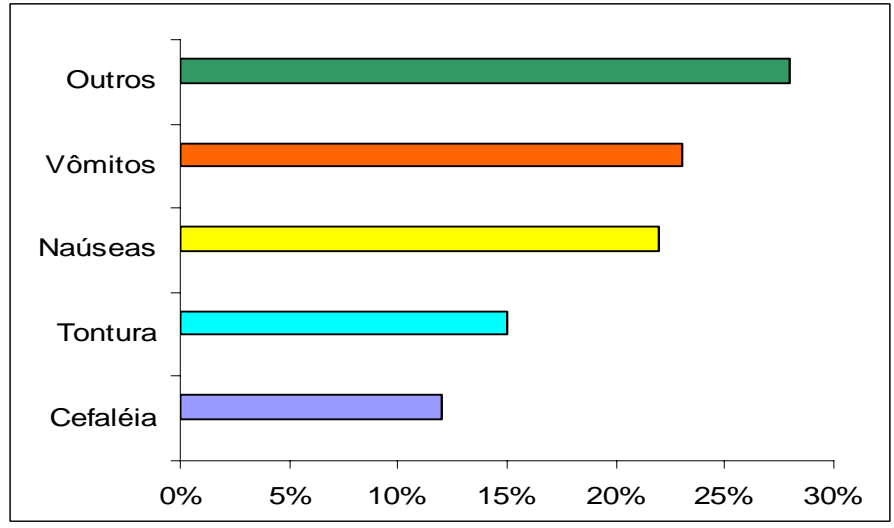

Figura 5: Distribuição das reações adversas entre os pacientes acompanhados no Hospital Gonzaguinha de Messejana.

As hipóteses diagnósticas mais comuns foram hipertensão arterial sistêmica (HAS) 42\% ( $n=1411$ ), gripes e resfriados $28 \%(n=941)$, crises asmáticas com 18\% e trauma $12 \%(n=403)$, Figura 6. 


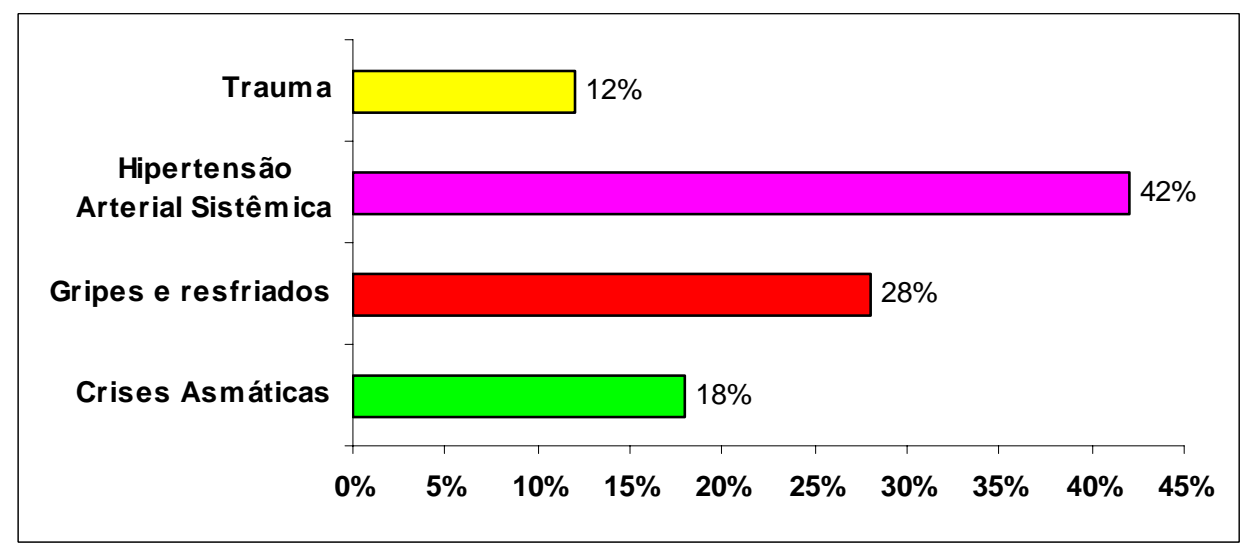

Figura 6: Principais hipóteses diagnósticas encontradas entre os pacientes acompanhados no Hospital Gonzaguinha de Messejana.

Dentre as outras hipóteses encontradas destacam-se cefaléia, gravidez, desnutrição, desidratação, neurocisticercose, câncer de mama, insuficiência cardíaca congestiva, dispnéia, hemorragia digestiva alta, hérnia discal, osteomielite de crânio, aneurisma, esclerose óssea, obesidade mórbida, politraumatismos, hidrocefalia e meningiomas. Os dez principais medicamentos mais prescritos na emergência foram dipirona, paracetamol, amoxicilina, captopril, sulfato ferroso, cefalexina, diclofenaco sódico, prednisona, salbultamol, sulfametoxazol + trimetroprima (Figura 7).

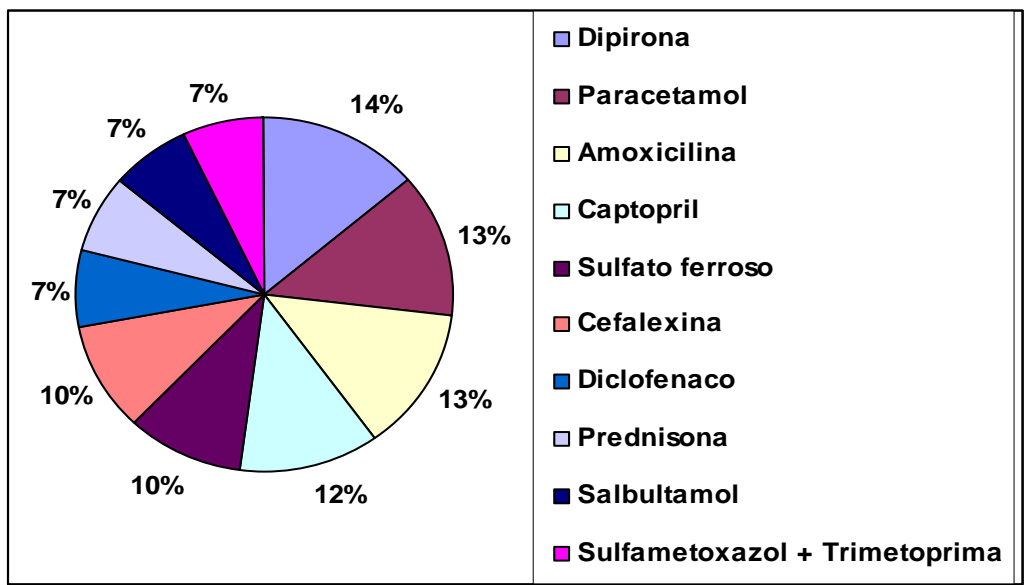

Figura 7: Principais medicamentos prescritos na emergência do Hospital Gonzaguinha de Messejana.

Na Figura 8 foram detectados um total de 586 PRM. O número de PRM por paciente foi em torno de 8 PRM/paciente. O PRM mais comum detectado nas duas unidades foi o PRM 6, 50\% $(n=293)$; seguido do PRM 4 , $19 \%(n=111)$, PRM 1, 5\% ( $n=29)$; PRM 2, 5\% $(n=29)$; PRM 3, 11\% $(n=64)$; e risco de PRM 5, 10\% $(n=59)$.

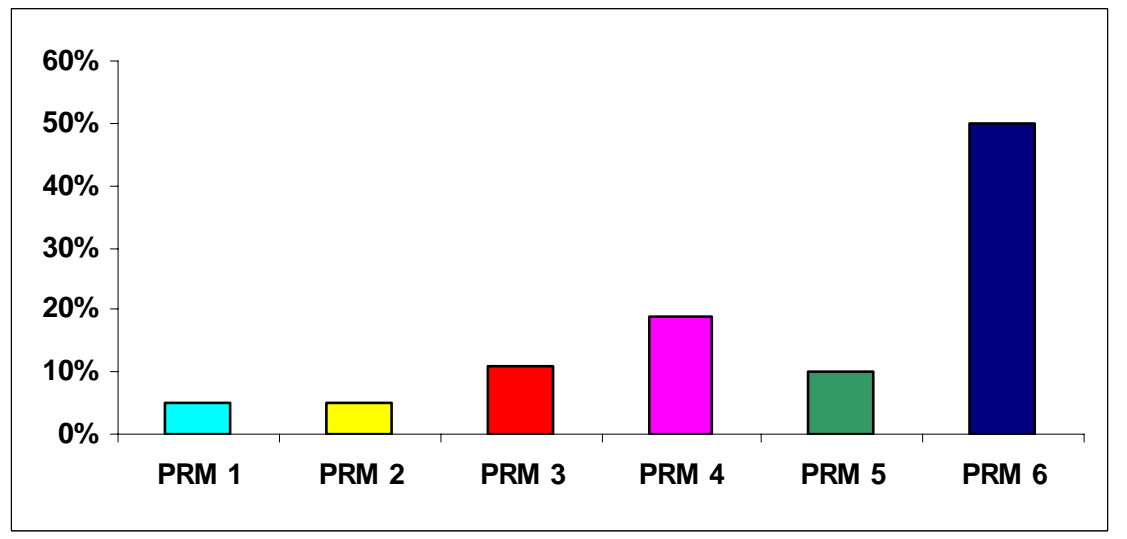

Figura 8: Total de problemas relacionados a medicamentos detectados na emergência do Hospital Gonzaguinha de Messejana. 
Os problemas relacionados à saúde mais comuns foram detectados em 780 pacientes, tais como: tabagismo 60,0\% $(n=468)$, etilismo 25\% $(n=195)$, insônia 10\% $(n=78)$ e dependência à cafeína 5\% $(n=39)$ (Figura 9).

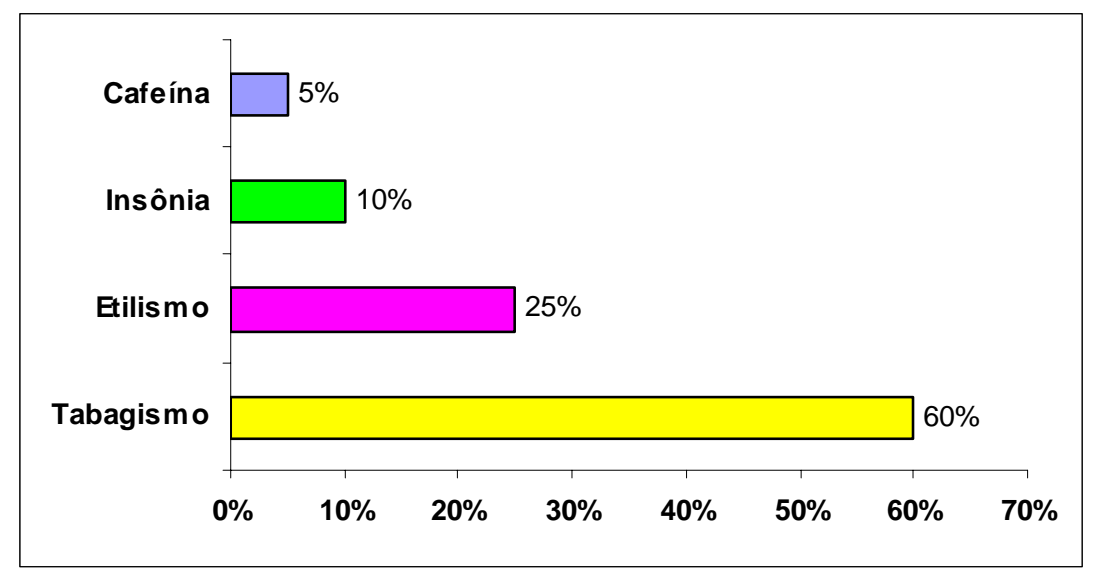

Figura 9: Principais problemas relacionados à saúde identificados entre os pacientes acompanhados no Hospital Gonzaguinha de Messejana.

O número de pacientes acompanhados é maior na emergência provavelmente devido à maior rotatividade nos leitos em conseqüência da internação temporária de pacientes provenientes de suas residências que apresentam agravamento do quadro clínico e quando melhoram, retornam as suas residências, da alta hospitalar, ou são transferidos para outra unidade de internação.

A faixa etária de maior prevalência entre os pacientes acompanhados foi de 31 à 50 anos (45\%) identificando ser a idade um fator de risco para o desenvolvimento de comorbidades (FAUCl, et al., 2002). A maior prevalência do sexo feminino (67\%) dentre os pacientes acompanhados pode estar correlacionada com a idade de maior prevalência, em que é possivelmente as pacientes encontram-se no período de menopausa. Esta etapa caracteriza-se pela redução na produção de hormônios provenientes do colesterol, estando este não consumido, aumentando sua concentração sérica e o risco de se aderir nos vasos sanguíneos e desencadear doenças cardiovasculares como trombose, infarto e também neurológicos, como o acidente vascular cerebral (FAUCl, et al., 2002). Os pacientes internados na emergência apresentaram maior número de problemas de saúde e doenças, uma vez que esta unidade destina-se a receber pacientes mais graves.

Foi observada correlação entre os problemas relacionados à saúde mais freqüentes entre pacientes e as hipóteses diagnósticas, pois alguns desses são fatores de risco para o desencadeamento das doenças apresentadas, como por exemplo, o tabagismo para acidente vascular cerebral e hipertensão arterial sistêmica, além do uso de contraceptivo associado ao tabagismo que também poder aumentar esse risco. Assim, como o etilismo pode causar hipertensão arterial sistêmica (FAUCI \& DPIRO, 2002; KODA-KIMBLE \& YOUNG, 2001) e seu agravamento pelo tabagismo (FAUCI \& DIPIRO, 2002; KODA-KIMBLE, et al., 2001). Vários efeitos neurológicos da hipertensão localizam-se no Sistema Nervoso Central. O infarto cerebral é secundário ao aumento de aterosclerose observado em pacientes hipertensos, enquanto a hemorragia cerebral decorre tanto da elevação da pressão arterial quanto do surgimento de microaneurismas vasculares cerebrais. Somente a idade e a pressão arterial sabidamente influenciam no surgimento de microaneurismas (DPIRO, et al., 2002; KODAKIMBLE \& YOUNG, 2001).

Pode-se observar que os medicamentos mais prescritos de acordo com o levantamento estão envolvidos com maior prevalência de PRM's 6, 5 e 4, problemas relacionados com efetividade e segurança ressaltando a necessidade de adoção de parâmetros monitorização desses aspectos na prática clínica, afim de previr complicações de interações e de reações adversas. Estes parâmetros não são habitualmente realizados na prática hospitalar possivelmente por limitação financeira, principalmente tratando-se de instituições públicas. Alguns dos medicamentos estão envolvidos com PRM 2, como exemplo o uso do paracetamol e da dipirona que demonstram a necessidade de se reavaliar o uso durante a internação, uma vez que apresenta grande potencial de interação medicamentosa e são prescritos por alguns profissionais de forma indiscriminada (THE UNITED STATES PHARMACOPOEIA, 2002).

O PRM de maior prevalência na unidade estudada foi o PRM 6, acredita-se que pela complexidade das doenças e pela necessidade de polimedicação durante o tratamento, os pacientes estejam sujeitos a sofrerem as conseqüências de interações medicamentosas que podem aumentar os efeitos farmacológicos ou exacerbar os 
efeitos tóxicos como identificado pelo PRM 6. A maioria deles apresenta-se como risco de desenvolver problema relacionado ao medicamento e para que se minimize a ocorrência destes é necessária a utilização de parâmetros de monitorização para que sejam realizados os ajustes terapêuticos necessários. A ocorrência de PRM 5 também foi significativa, mas se apresenta, em sua maioria, também como um risco para desenvolver PRM e para redução de ocorrência de reações adversas a medicamentos também é necessária a implantação de parâmetros de monitorização sugeridos na literatura (THE UNITED STATES PHARMACOPOEIA, 2002).

Outro PRM freqüente foi o PRM 4, em que o paciente não apresenta a resposta esperada ao tratamento farmacológico, seja por não assiduidade na administração das doses conforme a prescrição ou pela ocorrência de interações medicamentosas que podem reduzir a ação farmacológica, prejudicando os efeitos do medicamento e diminuindo a resposta do paciente. Nesse caso também seria necessário verificar a eficácia do tratamento por meio de parâmetros de monitorização do medicamento (THE UNITED STATES PHARMACOPOEIA, 2002).

\section{CONCLUSÃO}

A realização da Atenção Farmacêutica em hospitais é de difícil implantação devido à necessidade de discussões e convencimento da Diretoria Clínica e Administrativa quanto ao trabalho clínico, do farmacêutico através dos benefícios aos hospitais. Apesar disso, é de suma importância para a racionalização da terapia farmacológica e melhoria da qualidade de vida do paciente, além da redução de custos hospitalares, complementando o serviço administrativo prestado pela Farmácia Hospitalar através da integração do farmacêutico junto à equipe hospitalar com conhecimentos técnico-científicos. Talvez a dificuldade se explique pela gravidade dos quadros clínicos, complexidade das prescrições, dos procedimentos e desconhecimentos de aspectos técnicos pela equipe multiprofissional.

Alguns problemas relacionados ao medicamento podem ser detectados e resolvidos por meio da adoção de parâmetros de monitorização, como exames laboratoriais e resposta clínica do paciente. O farmacêutico é o profissional tecnicamente qualificado para aperfeiçoar a terapia medicamentosa prevenindo, detectando e corrigindo problemas relacionados aos medicamentos, tais como reações adversas, interações medicamentosas e incompatibilidades físico-químicas. Desta forma, garante-se o sucesso da terapia farmacológica, melhorando a qualidade de vida do paciente e pela redução de problemas relacionados à saúde, contribuindo dessa forma para redução dos custos assistenciais.

\section{REFERÊNCIAS BIBLIOGRÁFICAS}

ARANCIBIA, A. et al. Fundamentos de Farmácia Clínica, Santiago, Chile, Piedade, 1993.

BATES, D.W, et al. Drug and adverse drug reactions. How worried should we be? Journal of American Medical Association, v. 279, n.15, p. 1216-1217, 1998.

BARR, P.D. Hazards of modern diagnosis and therapy - the price we pay. Journal of American Medical Association, v. 159, n. 15, p. 1452-1456, 1955.

CIPOLLE, R.J. et al. El ejercicio de la Atencion Farmaceutica, Madrid: McGraw Hill. 2002.

$\mathrm{COUCH}$, N.P., et al. The high cost of low-frequency events. The anatomy and economics of surgical mishaps. The New England Journal of Medicine, v. 304, n.11, p. 634-637, 1981.

DIPIRO, J.T. et al. Pharmacotherapy - a patohphysiologic approach, New York: Appleton \& Lange, 2002.

FAUCI, A.S. et al. Harrison - tratado de Medicina Interna, Madri: Mc Graw Hill, 2002.

FERNANDEZ-LLIMÓS, F. et al. Problemas Relacionados com la Medicación. Conceptos y Sistemática de Clasificación, Pharm. Care Esp., v. 1, p. 279-288, 1999.

FLEMING, S.T. Complications, adverse events and iatrogenesis: classifications and quality of care measurement issues. Clinical Performance and Clinical Perfomance and Quality Health Care, v. 4, n. 3, p.137-147, 1996.

FONSECA, M.R.C.C., Fonseca, E., Bergsten-Mendes, G. Prevalência do uso de medicamentos na gravidez: uma abordagem farmacoepidemiológica, Saúde Pública, v. 36, n. 2, p. 205-212, 2002. 
GOMES, M.J.V.M., REIS, A.M.M. Ciências Farmacêuticas: uma abordagem em farmácia hospitalar, São Paulo: Editora Ateneu, 2001.

GRUPO DE INVESTIGACIÓN EN ATENCIÓN FARMACÉUTICA. Segundo Consenso de Granada sobre Problemas Relacionados com Medicamentos, Ars Pharmaceutica, v. 43, n. 3-4, p.175-184, 2002.

HEPLER, C.D., STRAND, L.M. Oportunidades y responsabilidades en la Atención Farmacéutica. Pharm Care Esp, V.1, n.1, p.35-47, 1999.

KODA-KIMBLE, M.A., YOUNG, L.Y. Applied therapeutics - the clinical use of drugs. Philadelphia: Lippincott Willians \& Wilkins, 2001.

MAIA-NETO, J.F. Farmácia hospitalar: um enfoque sistêmico. Brasília: Thesaurus, 1990.

MARIN, N., LUIZA, V.L., OSÓRIO-DE-CASTRO, C.G.S., MACHADO-DOS-SANTOS, S. Assistência farmacêutica para gerentes municipais, Rio de Janeiro: OPAS/OMS: 2003.

Ministério da Saúde. Guia Básico para Farmácia Hospitalar, BRASIL, 1994.

MONTRUCCHIO, D.P., ZANIN, S.M.W., MIGUEL, M.D., OYAKANA, C.N., OLIVEIRA, A.B. Obstáculos da Atenção Farmacêutica no Brasil, Revista Brasileira de Ciências Farmacêuticas, v. 41, n. 4, p. 409-413, 2005.

ORGANIZAÇÃO MUNDIAL DE SAÚDE. Informe de Tokio sobre el Papel del Farmacéutico em el Sistema de Atención de Salud. Ars. Pharm, v. 36, n. 1, p. 285-292, 1995.

ROSA, M.B., PERINI, E. Erros de medicação: quem foi? Rev. Assoc. Med. Bras. v. 49, n. 3, p. 335-341, 2003.

STELL, K, et al. Latrogenic illness on general medical service at a university hospital. Qual Saf Health Care, v. 13, n.4, p. 76-80, 2004.

THE UNITED STATES PHARMACOPOEIA: drug information for the heath care professional. Massachucetts: Micromedex Thomson Heathcare, 2002.

TORRES, R.M., OSÓRIO-DE-CASTRO, C.G.S., PEPE, V.L.E. Atividades da Farmácia Hospitalar Brasileira para com Pacientes Hospitalizados: uma revisão da literatura, Ciências \& Saúde Coletiva, v. 12, n. 4, p. 973-984, 2004. 